\title{
EFFICIENT ERROR CORRECTION SOLUTIONS FOR OFDM-BASED WIRELESS VIDEO
}

\author{
Mauro Lattuada, Renzo Posega, Marco Mattavelli, Daniel Mlynek
}

\author{
Ecole Polytechnique Fédérale de Lausanne (EPFL), Laboratoire de Traitement des Signaux (LTS3), \\ Batiment ELB, Station 11, CH-1015 Lausanne, Switzerland \\ E-mail: mauro.lattuada@epfl.ch
}

\begin{abstract}
This paper describes a new error correction scheme for flexible and robust high-throughput real time video transmission systems able to cope with difficult high mobility channels. The scheme is based on combining OFDM transmission with Turbo Coding and deep time interleaving. Simulations of different channel models and field test has shown that the provided solution provides relevant improvement versus DVB-T based solutions.
\end{abstract}

\section{INTRODUCTION}

The objective of this work is to study wireless digital video transmission system solutions achieving at the same time flexibility and robustness for high mobile applications. The OFDM modulation, for its intrinsic robustness to multipath fading has shown to be the best modulation choice for wireless digital video transmission. A simple evolution of nowadays well-established DVB-T [1] or ISDB-T [2] standard does not enable the development of an appropriate system for mobile applications. Several problems arise when trying to use transmission channels placed in higher frequency bands. The major problems are due to increased Doppler and phase noise impairments that can completely corrupt the performances of current receivers designed for less critical DVB-T mobility. New highly mobile applications require the development of appropriate OFDM processing architectures. Moreover, DVB-T standard modes are well suited for fixed bandwidth transmission to be compatible with existent analog broadcasting and cannot be easily modified to enable transmission into variable bandwidth channels. The available channel bandwidth in wireless digital video application can be very different. The most important goal of this work is the investigation of the possible improvements achievable by the Forward Error Correction (FEC) schemes combined with deep interleaving techniques applied to OFDM modulation in presence of deep fading impairments of the transmission channel. The objective is to achieve sufficient robustness for enabling a QoS for real time video contributions. While the digital television standards use a concatenated error correction scheme based on convolutional inner coder, in this work the investigated solution is based on a turbo inner coder. These codes offer theoretically better performances than convolutional codes, but the complexity and the computational requirements are higher. A concatenated turbo-block codes scheme solution has been investigated with the purpose of taking advantages of the improved error correction capabilities for reduced complexity portable applications. The concatenated scheme solution obtains very low BER and avoids error floors at low bit error rates.

The second element of the error correction scheme studied in this work is the channel interleaver used to average the channel fading in time and frequency. This mechanism is conceived to be adapted to the modulated bandwidth and is optimized for high throughput real time video. The proposed system architecture has been finally been implemented in FPGA. The application field of the proposed system is in the wireless transmission for digital television production like in the case of Digital News Gathering or the sport events production. Figure 1 shows a typical application of such system in the case of a bicycle race.

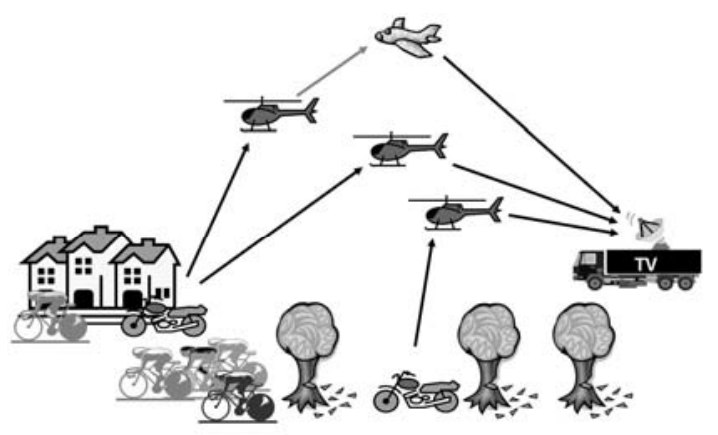

Figure 1: Bicycle race example.

\section{DVB-T}

The DVB-T modulation scheme was developed to guarantee transmission error ratios between $10^{-9}$ and $10^{-12}$. The DVB-T Forward Error Correction (FEC) system has been developed to work with fixed length MPEG2-TS (Transport Stream) packets. After a proper randomization to ensure the adequate binary transitions, the 188 bytes packets are Reed-Solomon $(204,188)$ coded. This outer coder adds 16 bytes and has a maximum correction capability of 8 bytes. After this, the outer interleaving, also called Forney convolution interleaving, is performed to scatter the errors at the reception side and so to make the outer coding more effective by shuffling the data bytes over 12 packets. The inner coder is a $1 / 2$ rate convolutional code $\left(\mathrm{G}_{1}=171_{\text {oct }} ; \mathrm{G}_{2}=133_{\text {oct }}\right)$ and performs 
different puncturing patterns to obtain different coding robustness $(\mathrm{R}=1 / 2 ; 2 / 3 ; 3 / 4 ; 5 / 6 ; 7 / 8)$. The inner interleaver consists in a bit-wise interleaving followed by a symbol interleaving. Both the bit-wise interleaver and the carrier interleaver processes are block-based and the block length depends on the OFDM mode $(2 \mathrm{~K} / 8 \mathrm{~K}$ carriers modes). The bit-wise interleaver, depending on the carrier modulation mode (QPSK, 16QAM, 64QAM) distributes the stream over different carriers to avoid selective fading corrupting consecutive message bits. The carrier interleaver then generates a permutation between carriers to spread in frequency the carriers over an OFDM symbol.

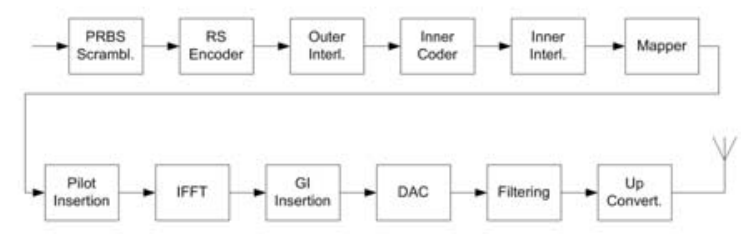

Figure 2: Standard DVB-T transmitter block diagram.

\section{FEC and System design}

\subsection{Turbo based concatenated FEC}

The Quasi Error Free performances $\left(\mathrm{BER}=10^{-12}\right)$ and the acceptable implementation complexity are contradictory requirements with a pure turbo code based error correction scheme. Turbo codes can provide such performances with a high number of iterations and block length and this impact directly with the memory and the processing power needed for real-time decoding. The solution developed and investigated in this work is to concatenate the turbo coder with a $(204,188)$ Reed-Solomon outer code to correct the residual errors in the stream. The concatenated RS outer coder avoids error floors at low bit error rates and increase the working range of the system with a rapid transition to the QEF condition. This scheme is similar to the proposed FEC for the DVB-S2 [3] where LDPC codes are concatenated with a $\mathrm{BCH}$ outer coder. The chosen inner coder is a CRSC (Circular Recursive Systematic Convolutional) turbo code [4] with code rate of $\mathrm{R}=1 / 3,2 / 5,1 / 2,2 / 3,3 / 4$.

Table 1: Outer interleaver parameters.

\begin{tabular}{|c|c|}
\hline Branches $(\boldsymbol{B})$ & 12 \\
\hline $\begin{array}{c}\text { FIFO depth } \\
\boldsymbol{b}=\boldsymbol{0} \div(\boldsymbol{B}-\mathbf{1})\end{array}$ & $17 \cdot b$ \\
\hline $\begin{array}{c}\text { Delay } \\
\text { (Number of packets) }\end{array}$ & 12 \\
\hline Total memory (bytes) & 2244 \\
\hline
\end{tabular}

The outer interleaver for the concatenated error correction scheme adopted is a convolutional interleaver based on TS packets. The statistics of the errors can vary with the channel characteristics so the convolutional interleaver has to perform good averaging over many TS packets to allow the outer decoder to be effective. At the reception side the bit-stream from the turbo decoder is firstly converted in byte-stream and the correct alignment is retrieved looking for the synchronization byte of the TS header. Table 1 reports the outer interleaver parameters.

\subsection{Time and Frequency Interleaving}

The phenomenon of multipath fading, operating to the OFDM modulated signals, groups the errors in burst and decreases the efficiency of the error correction. When an OFDM signal undergoes a multipath fading process some frequency ranges are highly attenuated thus several adjacent carriers are corrupted by additive noise. At the same way, shadow fading corrupts several consecutive OFDM symbols. Two possible strategies can be applied in the case of deep fading for OFDM symbols. The first is the frequency interleaving inside each OFDM symbol and the second, called time interleaving, among the OFDM symbol sequence.

The frequency interleaver takes the data at the output of the inner coder and has to interleave the bit stream to avoid consecutive bits to be mapped on the same carrier. This process can be divided in a bit-wise interleaver which interleaves consecutive bits into different mapped carriers and a carrier interleaver which generate a pseudo-random sequence for the carrier mapping. The two processes are block based and can be adapted to the number of modulated useful carriers of the OFDM symbol.

The time interleaver receives the modulated carriers data (2, 4 or 6 bit each data carrier) from the bit interleaver. The task of the time interleaver is to spread errors coming from shadow fading across several OFDM symbols to allow the channel coding mechanism to rebuild the corrupted data. The benefits of such system are proportional to the memory depth because of the greater averaging period. The time interleaver needs to work on a continuous data stream and treats a huge quantity of data. The interleaving technique applied must manage large memory and needs to access data by bursts. The structure of the interleaver is shown in Figure 3.

The main characteristic of this kind of interleaver is its inherent time-continuity. One faulty OFDM symbol is spread over B consecutive OFDM symbols and a group of length $E$ of faulty OFDM symbols is spread over $(B+E)$ symbols. The advantage of the convolutional interleaver is that it spreads time-consecutive errors evenly over the symbols. For our application the time interleaver works with blocks of 16 carriers called atoms. This simplifies the packaging of the data and improves the transfer bandwidth during the writing and reading process. Each OFDM symbol is divided into groups of carriers to vary the transmitted bandwidth. Each symbol is composed of maximum of $\mathrm{N}_{\mathrm{g}}=16$ groups composed of 96 useful carriers. The time interleaver works on variable length symbols having a number of atoms $\mathrm{N}_{\mathrm{a}}$ equal to:

$\mathrm{N}_{\mathrm{a}}=\mathrm{N}_{\mathrm{g}} \cdot 96 / 16=\mathrm{N}_{\mathrm{g}} \cdot 6$ 
The interleaving is done only among OFDM symbols so the number of branches needs to be $B \geq N_{a}$; $B$ needs also to be a multiple of $\mathrm{Na}$ to keep the correct atom position.

$$
\mathrm{B}=\mathrm{p} \cdot \mathrm{N}_{\mathrm{a}} \quad \mathrm{p}=1,2 \ldots
$$

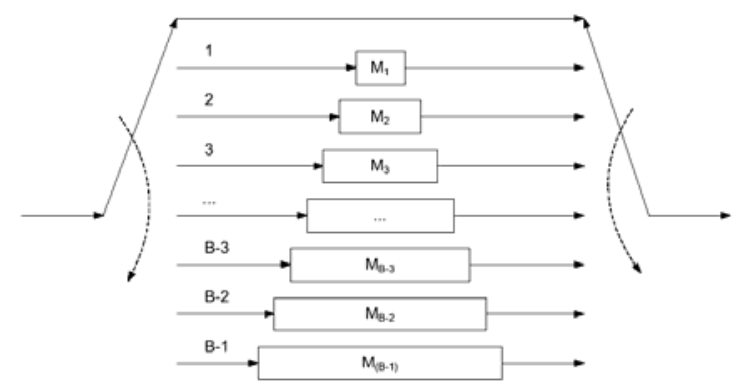

Figure 3: Time inteleaver.

Depending on the interleaving depth $\left(\mathrm{n} \cdot \mathrm{N}_{\mathrm{a}}\right)$ and the number of atoms, the FIFO sizes $\mathrm{M}_{\mathrm{b}}$ has to be chosen as:

$$
\mathrm{M}_{\mathrm{b}}=\mathrm{n} \cdot \mathrm{b} \cdot \mathrm{N}_{\mathrm{a}} \quad \mathrm{b}=1,2 \ldots,(\mathrm{B}-1)
$$

The delay will be equal to the number of symbol to store in the memory and equal to:

$$
\mathrm{D}=\mathrm{n} \cdot \mathrm{p} \cdot \mathrm{N}_{\mathrm{a}}=\mathrm{n} \cdot \mathrm{B}
$$

\subsection{System design}

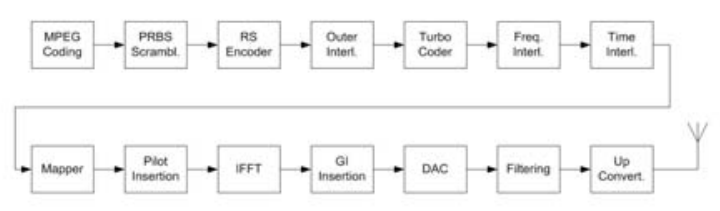

Figure 4: System block diagram.

The new developed system is flexible, and is able to adapt the modulation parameters depending on the application, the field conditions and the available frequencies. The spectrum is organized in blocks of carriers to achieve a variable bandwidth. Each symbol is organized in $\mathrm{N}_{\mathrm{g}}=16$ groups of 96 useful carriers and 12 pilot carriers for channel estimation and 1 signalization carrier. The pilot carriers are scattered inside the OFDM symbol to obtain a better frequency and time channel estimation and each block contains two fixed pilots. The time and frequency interleavers act only on the useful carriers of the OFDM symbol. This implies that the synchronization mechanism can not benefit of the time-symbol interleaving so it needs to support long time fading before falling into an unlocked status. Table 2 summarizes the main system parameters.

\section{Performances}

A complete system simulator has been developed to test the system performances. This $\mathrm{C}++$ test environment has been used to validate the modulation and demodulation algorithms and to provide the VHDL test vector for the FPGA implementation.

\subsection{Simulations results}

Table 3 shows the performances of the concatenated FEC compared to DVB-T. The table shows the required $\mathrm{E}_{\mathrm{b}} / \mathrm{No}$ for $\mathrm{BER}=2 \cdot 10^{-4}$ after inner coder and QEF after RS for

\begin{tabular}{|c|c|}
\hline $\begin{array}{l}\text { Useful channel } \\
\text { bandwidth (Bw) }\end{array}$ & $0.5-25 \mathrm{MHz}$ \\
\hline System frequency (fs) & $\begin{array}{l}12.5 \mathrm{MHz}(\mathrm{Bw}=0.5-10.6 \mathrm{MHz}) \\
25 \mathrm{MHz} \quad(\mathrm{Bw}=1-22,2 \mathrm{MHz})\end{array}$ \\
\hline Adjustable Steps $(\Delta B w)$ & $\begin{array}{l}0.66 \mathrm{MHz} @ 12.5 \mathrm{MHz} \\
1.31 \mathrm{MHz} @ 25 \mathrm{MHz} \\
\end{array}$ \\
\hline FFT size & $2 \mathrm{~K}$ \\
\hline $\begin{array}{l}\text { Maximum number of } \\
\text { modulated carriers (N) }\end{array}$ & 1745 \\
\hline $\begin{array}{l}\text { Maximum number of } \\
\text { useful carriers (Nu) }\end{array}$ & 1536 \\
\hline $\begin{array}{c}\text { Inter-carrier spacing } \\
\text { (df) }\end{array}$ & $\begin{array}{l}6,1 \mathrm{kHz} @ 12.5 \mathrm{MHz} \\
12.2 \mathrm{kHz} @ 25 \mathrm{MHz}\end{array}$ \\
\hline $\begin{array}{l}\text { Maximum number of } \\
\text { pilot carriers }\end{array}$ & 193 \\
\hline $\begin{array}{l}\text { Useful OFDM symbol } \\
\text { duration (Tu) } \\
\end{array}$ & $\begin{array}{l}163.84 \mu \mathrm{s} @ 12.5 \mathrm{MHz} \\
81 \mu \mathrm{s} @ 25 \mathrm{MHz} \\
\end{array}$ \\
\hline $\begin{array}{l}\text { Guard interval } \\
\text { duration }\end{array}$ & $1 / 4,1 / 8,1 / 16$ \\
\hline Constellation & QPSK, 16QAM, 64QAM \\
\hline Inner encoder & Turbo CRSC \\
\hline Inner encoding rate $(R)$ & $1 / 3,2 / 5,1 / 2,2 / 3,3 / 4$ \\
\hline Inner interleaving & Time and frequency \\
\hline $\begin{array}{c}\text { Maximum time } \\
\text { interleaver depth (Ti) }\end{array}$ & $\begin{array}{l}5.4 \mathrm{sec} \text { QPSK } \\
\text { 2,7 sec 16QAM } \\
1.3 \mathrm{sec} \text { 64QAM }\end{array}$ \\
\hline Outer interleaving & Convolutional on 12 packets \\
\hline Outer encoding & RS $(204,188)$ \\
\hline Useful bitrate & $\begin{array}{l}4,63 \div 37.9 \text { Mbits @ } 12.5 \mathrm{MHz} \\
9.26 \div 75,8 \text { Mbits @ } 25 \mathrm{MHz}\end{array}$ \\
\hline
\end{tabular}
the DVB-T standard.

Table 2: System parameters.

Table 3: Performances comparison for $\mathrm{BER}=10^{-4}$ at the output of the inner coder.

\begin{tabular}{|l|l|l|l|}
\hline & $\begin{array}{c}\text { Code } \\
\text { Rate }\end{array}$ & $\begin{array}{c}\text { DVB-T (CC) } \\
\mathbf{E}_{\mathbf{b}} / \mathbf{N o}(\mathbf{d B})\end{array}$ & $\begin{array}{c}\text { Turbo } \\
\mathbf{E}_{\mathbf{b}} / \mathbf{N o}(\mathbf{d B})\end{array}$ \\
\hline \multirow{3}{*}{ Gaussian } & $1 / 3$ & & 0.9 \\
\cline { 2 - 4 } & $2 / 5$ & & 1.2 \\
\cline { 2 - 4 } & $1 / 2$ & 3.35 & 1.35 \\
\hline \multirow{3}{*}{ Ricean } & $1 / 3$ & & 1.3 \\
\cline { 2 - 4 } & $2 / 5$ & & 1.6 \\
\cline { 2 - 4 } & $1 / 2$ & 3.95 & 2.1 \\
\hline \multirow{3}{*}{ Rayleigh } & $1 / 3$ & & 2.1 \\
\cline { 2 - 4 } & $2 / 5$ & & 2.8 \\
\cline { 2 - 4 } & $1 / 2$ & 5.75 & 3.95 \\
\hline
\end{tabular}

The gain obtained by using turbo codes results of about $2 \mathrm{~dB}$ for the Gaussian channel, $1.85 \mathrm{~dB}$ for the Ricean channel and $1.8 \mathrm{~dB}$ for the Rayleigh channel. Figure 5 
shows the BER evolution at the output of the turbo decoder for a small signal loss. The figure reports the evolution of the BER at the output of the inner coder for a signal loss of 100 symbols $(19.4 \mathrm{~ms})$ in the case of inner code $\mathrm{R}=1 / 3$, QPSK modulation, for $1.1 \mathrm{~dB} \mathrm{~Eb} / \mathrm{No}$ with Gaussian channel and time interleaving depth of 1920 symbols (384 ms).

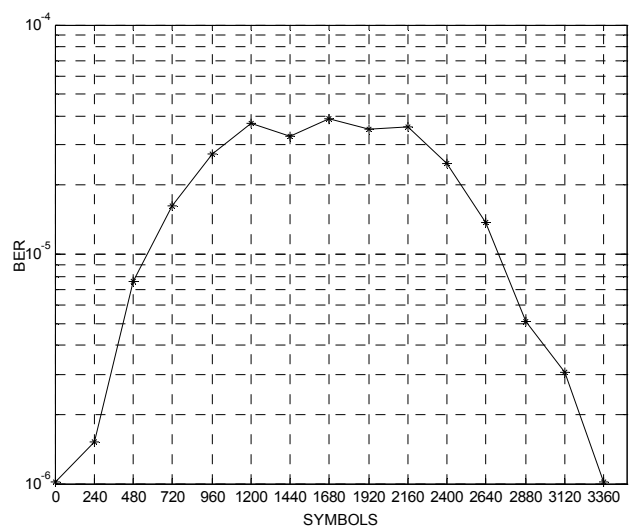

Figure 5: BER evolution at the output of the turbo decoder for a single failure point of 100 symbols and a time interleaver depth of 1920 symbols.

The graph clearly shows that the after turbo decoder the $\mathrm{QEF}$ threshold of BER $=2 \cdot 10^{-4}$ is not exceeded thus the deep fading event has been overcome without residual errors.

\subsection{FPGA implementation}

The modulator and the demodulator have been implemented on a Xilinx FPGAs platform. The modulator design results in a total equivalent gate count of $2.4 \mathrm{M}$ gates and works at $100 \mathrm{MHz}$. The demodulator is implemented on two FPGA's resulting in a total equivalent gate count of $10 \mathrm{M}$ gates working at $50 \mathrm{Mhz}$. The first FPGA receives the ADC data, control the AGC's and implements the channel estimation and compensation, the FFT and the demapping of the data carriers. The bit metrics are then output to the FEC section implemented on the second FPGA. This FPGA implements the time interleaver and controls a 256 Mbit SDRAM working at $100 \mathrm{MHz}$

\subsection{On field and implementation results}

The system performances have been compared with $8 \mathrm{MHz}$ bandwidth standard DVB-T demodulator. The transmitter works on $2.3 \mathrm{GHz}$ band with $10 \mathrm{dBm}$ emitted power. For QPSK modulation with $\mathrm{GI}=1 / 16$ and $\mathrm{R}=1 / 2$ the obtained performances are shown in Table 4. These results show the minimum power required at the input of the demodulator to obtain an error free image at the receiver. The implemented system has also been tested with a ground-plane transmission during some tests sessions.

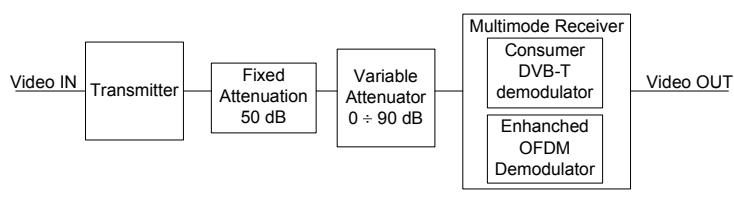

Figure 6: Implemented system test bench.

Table 4: Implemented system performances

\begin{tabular}{|l|l|}
\hline DVB-T & $-97 \mathrm{dBm}$ \\
\hline $\begin{array}{l}\text { Enhanced ODFM } \\
\text { 16 Iterations } \\
25 \text { ms Time interleaver }\end{array}$ & $-99 \mathrm{dBm}$ \\
\hline
\end{tabular}

During these tests the transmission system has been compared in real working condition with a limited power $(1 \mathrm{~W})$ in a very difficult environment (forest road). These tests show that a time interleaver between 100 and $800 \mathrm{~ms}$ improves the robustness of the link and allows the receiver to obtain a high quality image compared to the standard DVB-T transmission.

\section{CONCLUSION}

This work shows that a robust error correction system for high quality video mobile terminals based on turbo coding and time interleaving can improve the mobile performances of OFDM systems. The robustness of the system resides in a wide error spreading associated to a strong error correction system capable to cancel the impairments of the channel. The turbo based concatenated error correction demonstrate to be a good solution for QEF performances in mobile channels. The simulations show that the time interleaver is a very effective tool to successfully overcome shadow fading events. The experimental performances of the systems during field test confirm that the proposed error correction techniques can be really used to improve the performances of DVB-T in highly mobile applications.

\section{REFERENCES}

[1] Digital Video Broadcasting: Framing Structure, channel coding and modulation for digital terrestrial television (DVB-T). ETSI EN 300744 v1.4.1(200101)

[2] Terrestrial Integrated Services Digital Broadcasting (ISDB-T) Specifications for channel coding, Framing structure and modulation, ARIB, September1998.

[3] Digital Video Broadcasting (DVB-S2); Second generation framing structure, channel coding and modulation systems for Broadcasting, Interactive Services, News Gathering and other broadband satellite applications. Draft ETSI EN 302307 V1.1.1 (2004-06)

[4] D. Gnaedig, E. Boutillon, V. C. Gaudet, M. Jézéquel, P. G. Gulak, "On Multiple Slice Turbo Codes", in Proc. International Symposium on Turbo Codes and Related Topics, Brest, pp. 343-346, Sept. 2003 